ENCYCLOPEDDIE Encyclopédie berbère

BERBERE

$6 \mid 1989$

6 | Antilopes - Arzuges

\title{
Architecture berbère
}

L. Golvin

\section{OpenEdition}

Journals

Édition électronique

URL : http://journals.openedition.org/encyclopedieberbere/2582

DOI : 10.4000/encyclopedieberbere.2582

ISSN : 2262-7197

\section{Éditeur}

Peeters Publishers

\section{Édition imprimée}

Date de publication : 1 janvier 1989

Pagination : 865-877

ISBN : 2-85744-324-2

ISSN : 1015-7344

Référence électronique

L. Golvin, «Architecture berbère », Encyclopédie berbère [En ligne], 6 | 1989, document A264, mis en ligne le 01 décembre 2012, consulté le 12 octobre 2020. URL : http://journals.openedition.org/ encyclopedieberbere/2582; DOI : https://doi.org/10.4000/encyclopedieberbere.2582

Ce document a été généré automatiquement le 12 octobre 2020

(c) Tous droits réservés 


\section{Architecture berbère}

\section{Golvin}

1 On ne peut nier l'existence d'une architecture berbère, un art qui présente de multiples facettes qui a pu subir quelques contagions, mais un art qui, des côtes de l'Atlantique à la Libye, affiche sa parenté, celle d'une civilisation que le temps ni les aléas de l'Histoire n'ont pas condamnée ; étonnante pérennité qui a subi, à toutes les époques, l'assaut de l'étranger sans se laisser emporter par le vent de l'Histoire.

2 Cet art ne se manifeste pas par des monuments prestigieux aux imposantes dimensions et au décor somptueux. Il n'utilise pas de matériaux nobles tels que le marbre, la pierre de taille, le bronze, le cuivre, voire l'or ou la céramique émaillée, il n'a ni la puissance des réalisations antiques, ni la hardiesse des cathédrales médiévales. S'il fallait définir en un mot cette architecture, nous dirions qu'elle est essentiellement familiale, ce terme signifiant aussi bien la famille nucléaire que la tribu. Point de réalisations royales qui mettent en œuvre des centaines d'esclaves et qui utilisent les techniques les plus perfectionnées. On utilise le matériau trouvé sur place, la terre, qui, deviendra le pisé, les cailloux, ramassés sur la hamada ou sur les pentes de la montagne, le bois des palmiers, des cèdres ou des pins des forêts du Haut ou du Moyen Atlas. Certes, l'architecture religieuse subi les contraintes imposées par la tradition islamique, mais elle marque son originalité dans des formes très particulières, pratiquement inconnues ailleurs, telles les mosquées du Mzab ou du Sud Algérien ; mais, les réalisations les plus spectaculaires sont sans doute ces grands palais du Haut Atlas marocain ou ces greniers-citadelles des ksours, de l'Aurès ou du Sud Tunisien. 


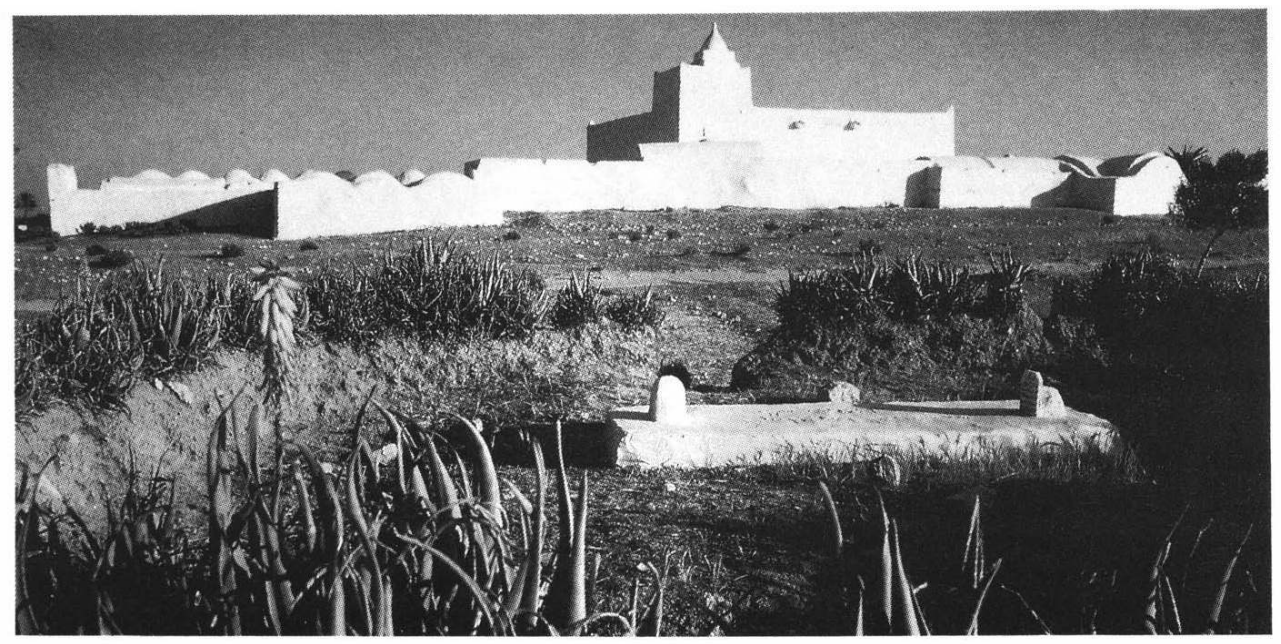

Mosquée de Beni Isguen (Mzab) (plan Y. Bonète).

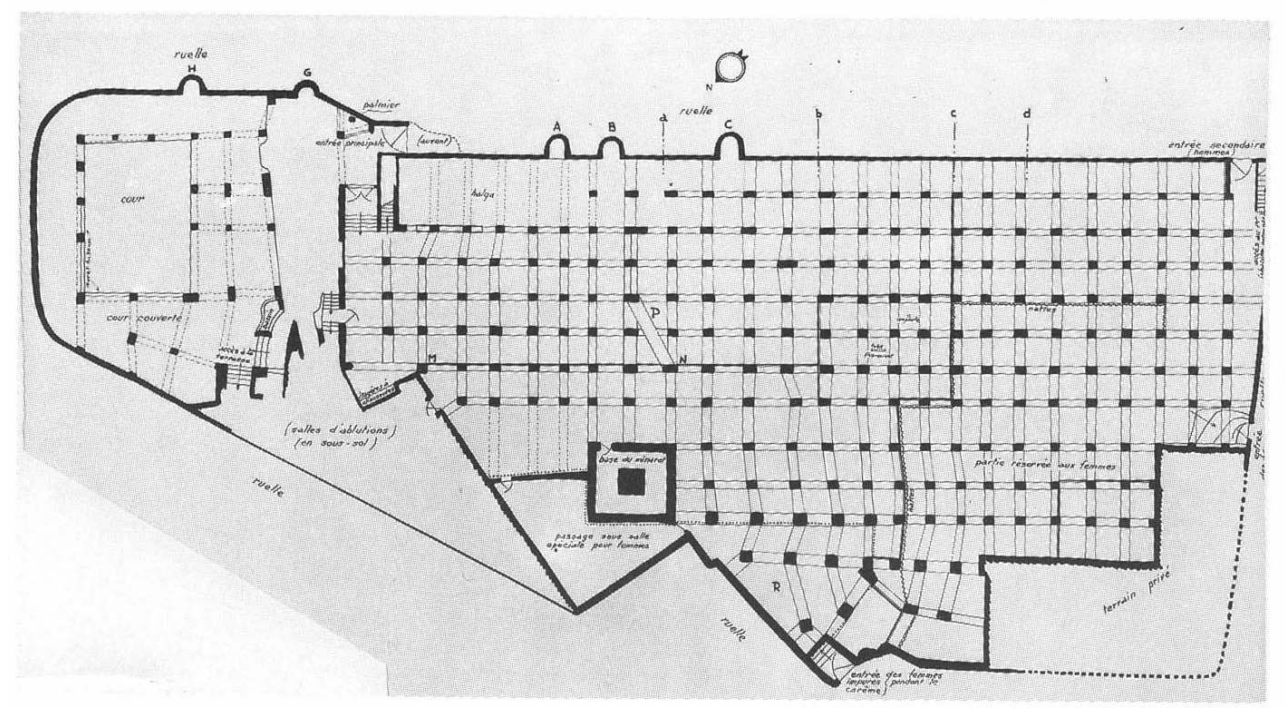

\section{L'art religieux}

Nous ne retiendrons que les monuments les plus typiques.

Les mosquées de Djerba: dispersées dans la palmeraie, les anciennes mosquées de Djerba (ancienneté toute relative) ont subi l'influence de la Libye toute proche; elles en ont retenu la forme des minarets ronds effilés, peu élevés, coiffés d'un dôme, les salles de prière trapues, couvertes de coupoles, les cours exiguës. Construites en pierre et blanchies à la chaux, elles ne sont sans doute pas vraiment représentatives de l'art berbère; on y trouve des influences ottomanes certaines, celles surtout des provinces autrefois rattachées à la Sublime Porte et plus précisément celles des villages du HautNil, de la Cyrénaïque et de la Tripolitaine.

Les mosquées du Mzab: Beaucoup plus étonnantes sont les mosquées du Mzab juchées au sommet de la cité, leur minaret en tronc de pyramide effilé dressé sur le ciel. Le plan complexe de la Grande Mosquée de Ghardaïa atteste de nombreux remaniements et 
plusieurs agrandissements; tous les murs sont gauches, probablement en raison de la déclivité du terrain; les alignements de piliers dans la salle de prière sont très rarement assurés, la cour, réduite et amputée par trois nefs est de guingois ; les piliers sont reliés par des arcades grossièrement façonnées disposées dans le sens nord-sud. Pas de décor. Les matériaux sont le pisé et la brique, la couverture est composée de troncs de palmiers supports de terrasses, mais on rencontre également des voûtes d'arêtes, voire des plafonds de pierres plates. Une section est réservée aux Ikhouān, une autre est destinée aux femmes. Le minaret se trouve en partie dans l'oratoire ; enduite de multiples couches de chaux superposées, la tour se dresse, carrée, s'effilant vers le haut, terminée par quatre pointes angulaires. A ses côtés existe toujours la petite cour en tronc de pyramide, sans doute aussi âgée que la mosquée. Deux mihrâb-s ouvrent sur la cour... Plus réguliers se présentent les plans des autres mosquées principales de la pentapole : Celui de Beni Isguen, où l'on décèle divers agrandissements, se développe en largeur sur environ 63 mètres et en profondeur sur 26 mètres; son minaret assez semblable à celui de Ghardaïa se trouve en partie dans l'oratoire, bordé au nord et à l'ouest par une petite cour, les salles d'ablution sont en sous-sol. Plus petites sont les mosquées de Mélika, de Bou Noura, d'El-Atteuf. Outre ces oratoires, il faut mentionner la très belle mosquée dite de Sidi Brahim, aux angles arrondis et aux murs percés de lucarnes et également la mosquée funéraire du cimetière de Beni Izguen avec ses multiples arcades ; puis les oratoires éloignés dans la palmeraie de Beni Izguen ou d'ElAtteuf. Toutes ces curieuses constructions, blanchies à la chaux, avec leurs arrondis, leurs ruptures de volumes, leurs ouvertures distribuées un peu n'importe comment, évoquent une architecture révolutionnaire et l'on a pas manqué d'y voir des créations d'avant-garde du type « Le Corbusier ».

Ghardaïa (Mzab), vue générale (photo L. Golvin).

Image 3.png

Dans les confins du Sahara algérien, il faut citer les beaux minarets de terre de Bou Chagroun, dans les Zibans, ceux de Sidi Khalif, de Doucen (Ziban), ce dernier très élevé, et en tronc de pyramide qui évoque celui de terre, aujourd'hui disparu, d'Ouled Djellal, la curieuse tour d'El-Bordj qui, sur une base carrée en briques cuites, possède un fût prismatique prolongé d'un cylindre porteur d'un lan-terneau, le minaret de Temmacin, l'un des plus beau du Sud Algérien offre une tour carrée construite en briques appareillées en réseaux losangés apparemment inspirés par le Maroc.

On doit également citer les mosquées du Souf aux multiples coupoles grises boursouflées, semblables à des nids d'hirondelles, ou lisses et blanchies à la chaux, leurs tours peu élevées, carrées, leurs cours étroites où les élèves se groupaient autour du mu'allem, à l'ombre du minaret, aux heures chaudes de l'été.

7 A Melika, on notera les étranges tombeaux de Sidi Aïssa dressant sur le ciel des espèces de cierges arrondis enduits de multiples couches de chaux et, à Metlili des Chaamba, de pittoresques coupoles à pinacles.

8 Cette architecture religieuse n'a nulle part son équivalent en pays arabe et, si l'on veut, çà et là, déceler des emprunts à des types connus au Maghrib, elle a suffisamment de caractère pour se distinguer nettement de l'art dit arabe, celui de l'Ifrîqiya influencé par l'Orient ou celui du Maghrib al-Aqșâ marqué par l'art de l'Espagne musulmane. Il est à noter que les grandes dynasties berbères qui régnèrent sur l'Afrique du Nord, Zîrides, Ḥammādides, Almoravides, Almohades, Ḥafșides, Marinides et Abd al-Wādides, 
ont adopté d'emblée et sans exception l'art de l'Orient ou celui de l'Occident hispanomusulman, tandis que se perpétuait, au Sahara, une architecture religieuse rurale sans doute définie dès le haut Moyen Age.

\section{L'architecture civile}

9 Nous ne savons rien de ce que furent les demeures de Tâhart, mais des fouilles archéologiques conduites à Sadrata par Blanchet en 1908 puis par Marguerite Van Berchem en 1951-52, nous ont révélé des demeures remarquables influencées apparemment par l'art de Samarra. Les murs étaient partiellement recouverts de parements de stucs, plâtre local chargé de sable, défoncés d'un décor essentiellement géométrique où l'on trouve, entre autres, des figures en forme de rosaces rappelant étrangement parfois les sculptures des coffres kabyles.

Mais, dès que l'on évoque l'architecture domestique, c'est vers le Haut Atlas marocain que le regard se porte, vers ces hautes qasba-s de terre rouge avec leurs tours carrées hérissées de merlons en dents de scie, leurs belvédères et leurs décors de chevrons; architecture de montagne que l'on ne peut s'empêcher de comparer à celle du Yémen (plus particulièrement à celle de Saada et de sa région ou à celle de Shibām (Yémen du sud). On songe également aux qș̂ur-s (Ksours) des vallées du Zîz et du Dadès, villages tribaux resserrés entre les hauts murs de remparts solidement construits en pisé rouge, ces tiүrhemt également en pisé de pierraille, aux murs extérieurs défoncés de décors losangés; avec leurs tours carrées d'angle, à terrasse débordante, et leurs merlons en dents de scie, leurs meurtrières, leurs šubbâk-s (Iglioua Sud, Tamesla des Aït Ouarzazate, Ouled Yahya, Aït Youssef, Imgoum, Ait Ougoudid, etc.). Architecture puissante dont l'originalté est évidente. La couverture est la terrasse de terre battue supportée par des lattis de branchages et de broussailles, parfois de lattes disposées en épi reposant sur des poutres en tronc d'arbre à peine dégrossi. L'un des plus beaux ensembles est sans doute la qașba de Ouarzazate. Le matériau est le pisé et la brique crue recouverte d'un enduit de chaux et de plâtre. A la fois résidence princière du Glaoui, elle était un véritable fortin. La qaṣba se hérisse de tours en tronc de pyramide crénelées de merlons, elle s'élève sur trois niveaux, voire davantage. Le mur de façade se défonce d'ouvertures étroites au rez-de-chaussée, véritables meurtrières ; mais, plus haut, on ne craint pas les fenêtres largement ouvertes sur l'extérieur, protégées par des grilles de fer forgé. Les murs s'ornent de défoncements en niches verticales prolongées, au sommet, par des meurtrières. Plus haut, on peut voir un édicule saillant qui permet de surveiller la base de l'édifice et plus particulièrement la porte d'entrée. Au Yémen, de tels édicules saillants sont appelés šubbâk-s. Ces formes proéminentes apparaissent parfois comme de véritables moucharabiés (mašrabiya) ouverts par de larges fenêtres sur l'extérieur et supportés par des jambes de force. 


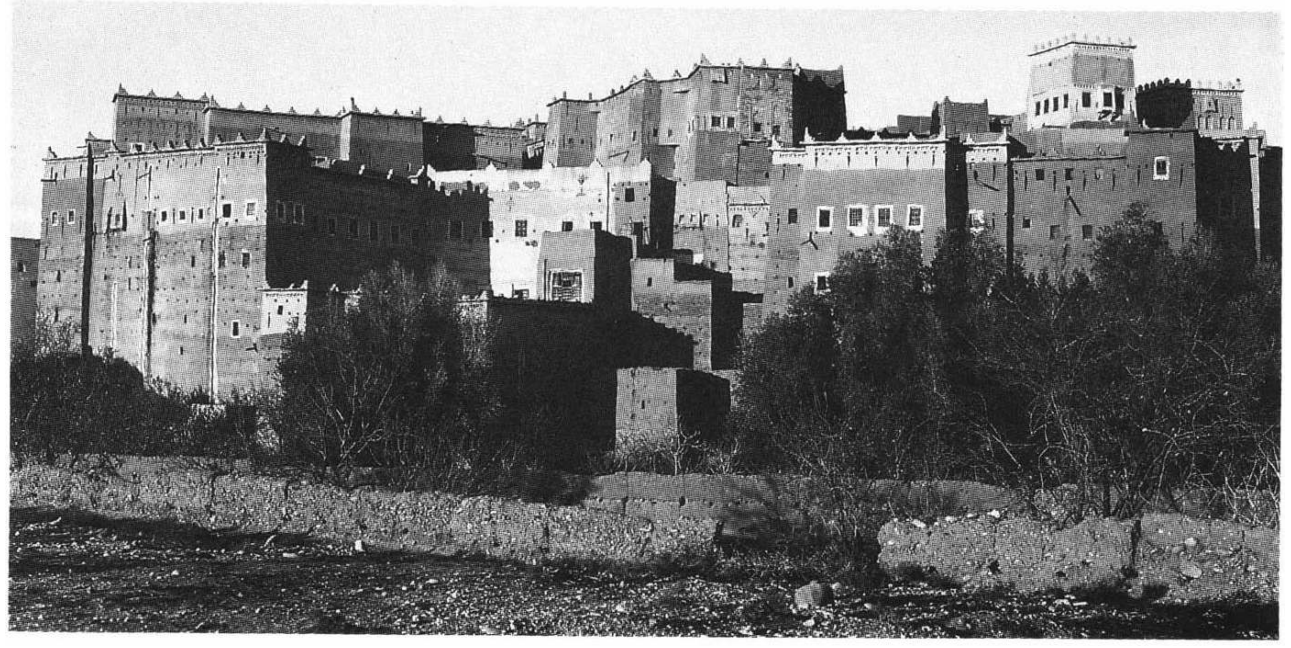

Qasba de Ouarzazate, partie haute (photo L. Golvin).

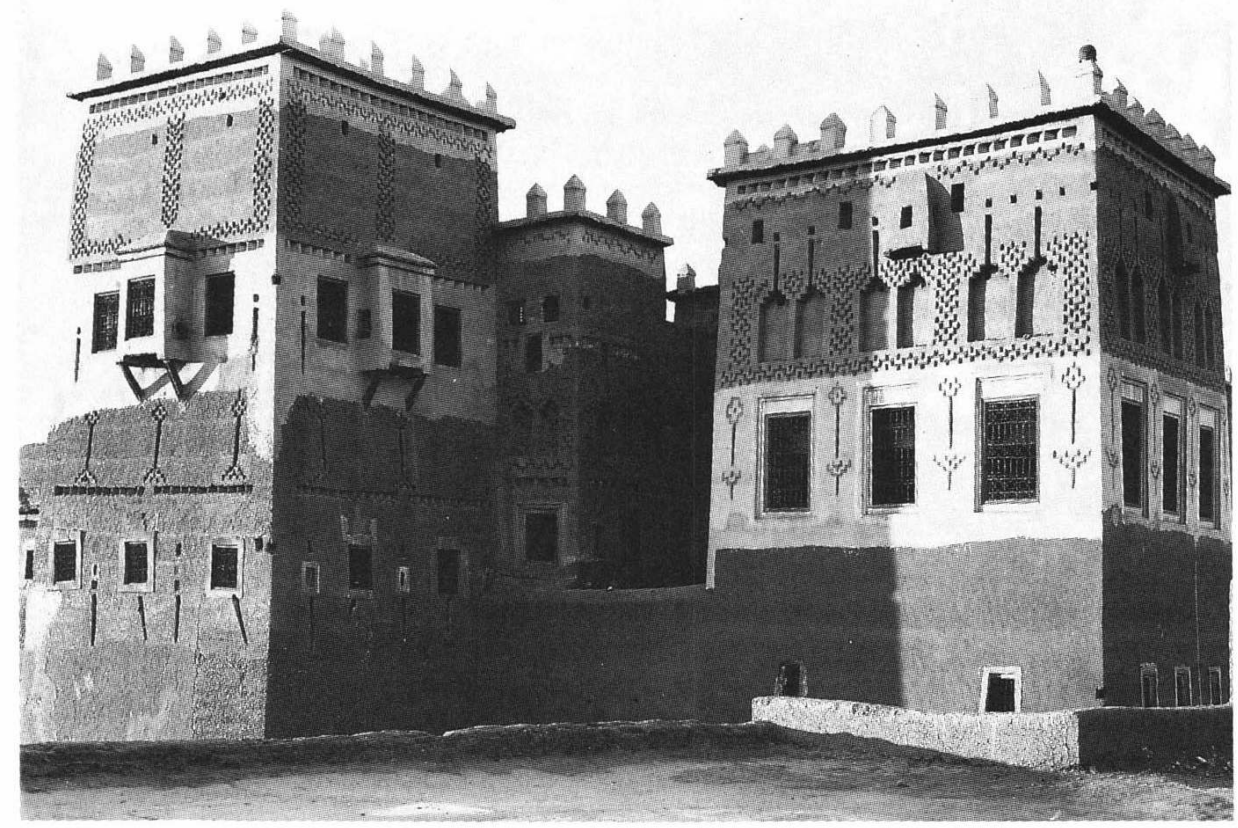

11 Des badigeons de chaux soulignent les parties nobles de la demeure, endroits où réside la famille. Les curieux décors de façade procèdent d'effets de briques disposées en quinconce, ils bordent les grandes fenêtres ou bien ils dessinent des chevrons, des frises à dents d'engrenage, des losanges, des triangles, ils tapissent tout un étage.

\section{La maison kabyle}

12 Son originalité réside surtout dans son mode de couverture qui est à deux pentes et à tuiles demi-rondes. Elle utilise essentiellement la pierre (un moellon dégrossi). Très fruste, elle comporte une seule pièce d'habitation (aguns ou tiүeryert) dans le sol de laquelle se creuse le foyer (kanun) et le mortier où se fixe le moulin à bras. 
13 Au mur, à gauche de l'entrée, une banquette est adossée, (iqedar)percée de niches où l'on range la vaisselle. En face est un mur de refend, le tadequouant peu élevé et percé d'ouvertures carrées, il supporte un plancher au-dessus duquel se trouve un grenier qui sert également de lieu de couchage et de réserves alimentaires contenues dans des ikufan (sing. akufi*); on entrepose là également les instruments aratoires. Sous le plancher se trouve l'étable. Les maisons se resserrent les unes contre les autres sur le point le plus élevé du site (taurirt) selon un plan rayonnant et des assises concentriques (Aït Larba) ou sur un plateau élevé (aguni) où le groupement est plus libre (Beni Yenni).

\section{La maison aurasienne}

14 Elle est en pierres (moellons dégrossis noyés dans un mortier de glaise avec chaînage de boulins horizontaux); elle est couverte d'une terrasse en pente, débordant sur les murs : amalgame de pierraille et de glaise tassées, reposant sur des lits de fascines. De gros galets posés aux extrémités de cette couverture la portègent des bourrasques. Sur l'extérieur, les murs sont percés de petites ouvertures triangulaires alignées et de fenêtres carrées, parfois également de lucarnes hexagonales à rayons en pierre taillée en fuseau. On voit encore des ouvertures allongées horizontalement, garnies de pierres en fuseau disposées en zigzag. La porte, épaisse, à panneaux verticaux mal dégrossis, s'orne de décors sculptés : chevrons, hexagones, triangles opposés : la serrure est en bois dur à tirette et à chevillettes. Des piliers en tronc de cèdre, au centre de l'unique pièce, supportent des poutres (troncs d'arbre à peine dégrossis) sur lesquelles s'appuient des solives faites de branches plus petites. Des fascines de branchages y sont couchées. Certaines maisons comportent deux niveaux : le rez-de-chaussée est destiné aux animaux : moutons, chévres, ânes, l'étage sert d'habitation.

\section{La maison de l'Anti-Atlas marocain}

Dans les tribus Ameln et Ida ou Semlal de l'Anti-Atlas, la maison se nomme tigemmi; généralement il s'agit d'un bâtiment carré d'un ou deux étages, parfois trois, de forme tronconique, couvert d'une terrasse. Sur l'une des façades, légèrement en saillie, se dresse un porche sur toute la hauteur de l'édifice, sorte de niche à fond plat couronnée d'un arc brisé outrepassé historié d'un décor à registres horizontaux superposés. En bas est la porte (taggurt) à un battant clouté sculpté d'arcades outrepassées et d'un quadrillage ; elle s'orne encore de belles pentures en fer forgé ainsi que de heurtoirs ; la serrure est en bois dur, avec tirette et chevillettes. Au-dessus se répartissent des registres décorés et des ouvertures carrées; les éléments du décor sont des pierres plates (ikwafaf) scellées de chant et formant des bandeaux de damiers ou de chevrons. Les murs (agrab) sont en moellons (azrû) jointoyés d'un mortier de graviers et de terre grise (akal). Les parois sont nues extérieurement (chez les Ida u Semlal) ou enduits de chaux (chez les Ameln). 
Ksour de Tamesla (Maroc), photo L. Golvin.

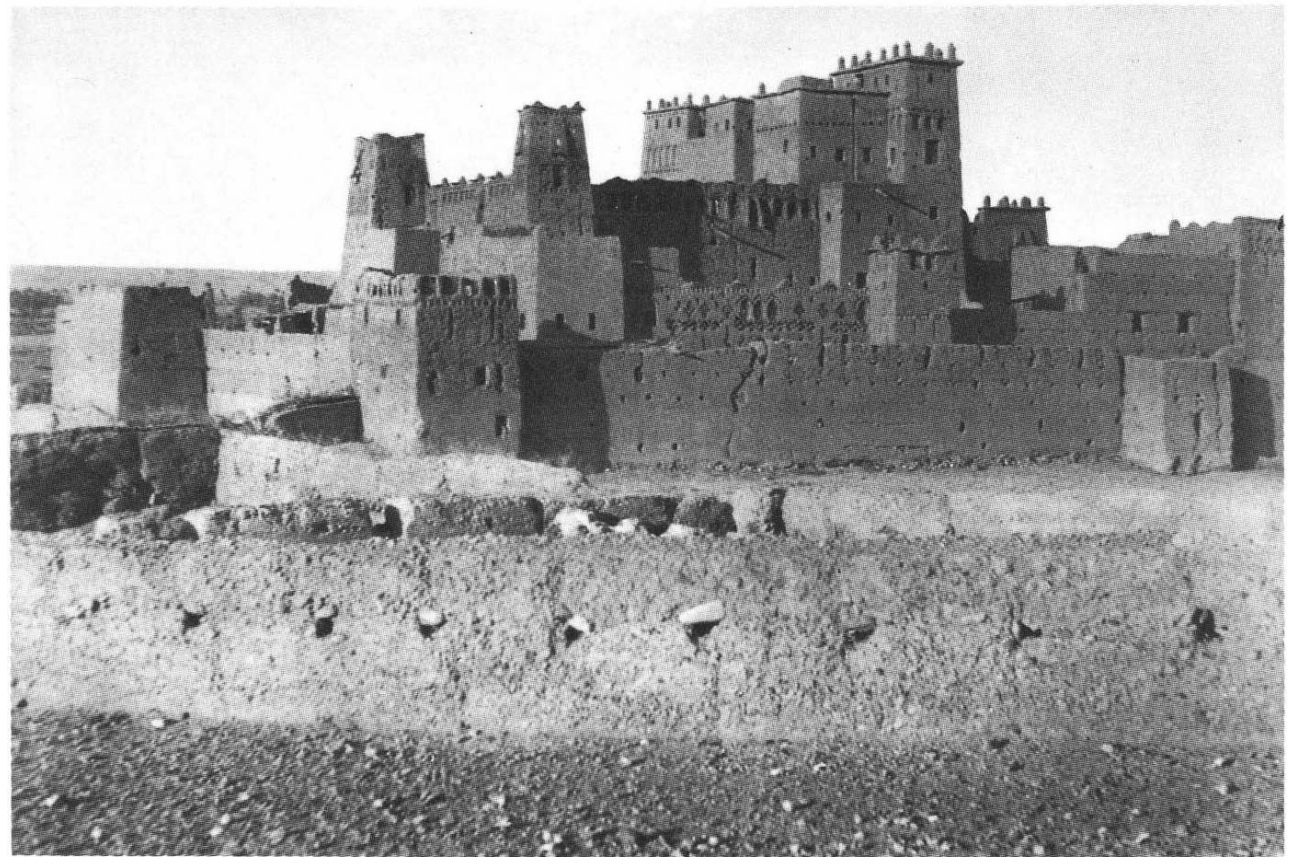

Une Qasba à Tilouet (photo H. Terrasse).

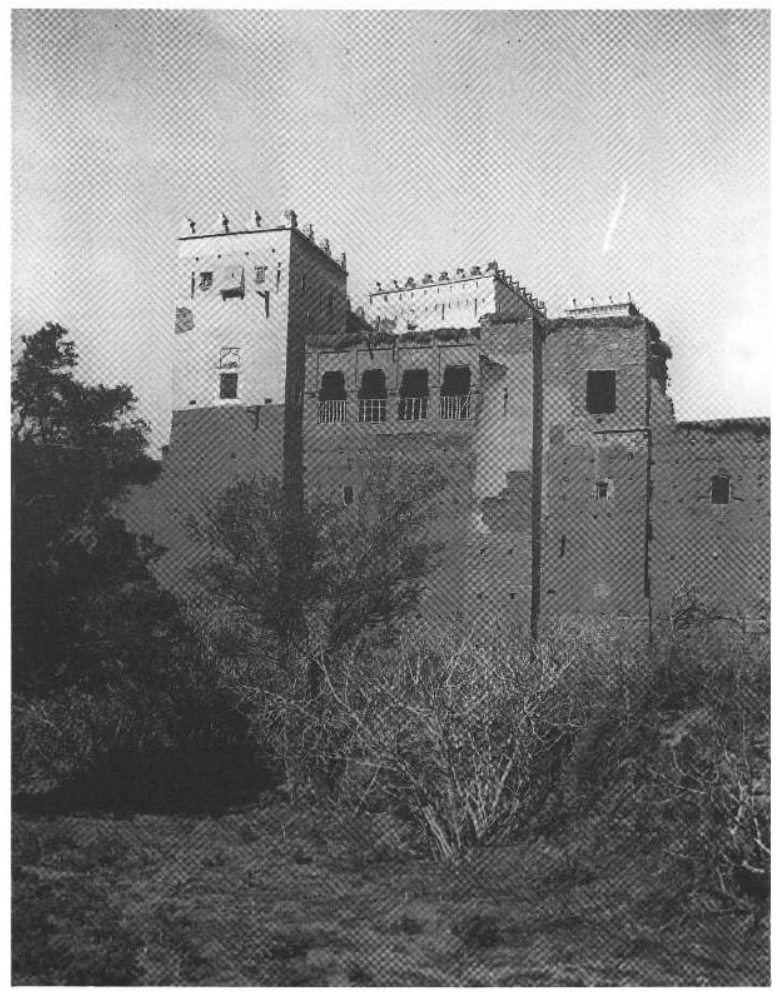

16 A l'extérieur quatre piliers (anebadad) déterminent un puits de lumière et d'aération qui débouche sur la terrasse. De ces piliers partent, vers les murs d'enceinte, des poutres (troncs d'amandiers ou de palmiers) supports des parquets de la terrasse. 
17 L'accès à l'étage s'effectue par des plans inclinés. Le rez-de-chaussée est occupé par des écuries (asarag) tandis que l'étage est le lieu d'habitation. Les murs de refend sont en briques crues.

Les plafonds se composent de solives parallèles supportées par des madriers transversaux sur lesquels on natte des tiges de laurier rose teintes. Dans un angle de la bâtisse se dresse une sorte de tour en saillie, crénelée de merlons en dents de scie.

Ces maisons se resserrent en petites agglomérations (muda') au pied de hautes falaises.

\section{La maison mozabite}

20 A Ghardhaïa, la maison (taddert pl. tiddart) se présente extérieurement sous la forme d'une façade nue défoncée de trois ouvertures: la porte (taurt, pl. tiuira) surmontée d'une lucarne (ullun pl. illunen) et d'un trou carré, à gauche de la porte, par lequel on peut actionner la fermeture (serrure en bois dur à tirette et chevillettes manœuvrées à l'aide d'une clef spéciale). L'entrée indirecte (imi) comprend un couloir tournant à angle droit sur le patio (ammas) en partie couvert, ne laissant au centre qu'un carré de ciel fermé d'une grille de fer. Ce patio, où, la plupart du temps, se tiennent les femmes, comporte de nombreuses niches murales carrées, un coin cuisine, surmonté d'étagères superposées (maçonnées) pour le rangement des ustensiles de ménage des produits d'usage courant, un autre coin (tahaja) est occupé par le métier à tisser.

21 Sur ce patio central s'ouvrent, au rez-de-chaussée, plusieurs chambres (tazka, pl. tizkaui) dont l'une, appelée tiziffri ne possède qu'une ouverture béante, elle sert de salle de prière; les autres pièces sont à usages multiples; des latrines se trouvent au fond du couloir.

22 Une cave (baju, pl. ibuja), en sous-sol, est en temps normal destinée à la conservation des denrées telles que les dattes, mais elle offre, en été, un abri appréciable contre les fortes chaleurs à ceux dont les occupations ou les ressources ne permettent pas de disposer d'une résidence d'été dans la palmeraie.

A l'étage, on trouve une galerie d'arcades sur piliers sur deux côtés, déterminant deux portiques (ikumar). Au centre est le patio supérieur percé d'un trou carré et grillé déjà évoqué. Une chambre ouvre sur le patio, elle est dotée d'un réduit toilette (azru uaman) et bordée de latrines. C'est la chambre d'hôte ; une autre pièce donne sur la galerie ; elle sert souvent de réserve à provisions (h'ujerete).

Des rondins de bois saillants servent de porte-manteaux. La porte d'entrée unique, lourde et massive, s'applique dans un cadre à piédroits supportant un linteau soulagé par un arc de décharge. Elle se compose de planches de palmier assemblées, renforcées d'un bandeau horizontal décoré qui supporte un anneau de fer forgé. Une de ces planches verticales forme gond (ided) par deux appendices saillant en haut et en bas. Côté dos, les planches sont maintenues par trois traverses sculptées de petits triangles; celle du milieu supporte un anneau métallique (tisel-sel) servant à tirer le battant. La fermeture (duart, pl. tidduarin) est en bois dur ; on ne peut l'actionner que de l'intérieur, elle se compose d'un tirant et de chevillettes de bois descendant dans des encoches du pène. On ne peut déverrouiller qu'à l'aide d'une clef spéciale, également en bois, munie de petits tenons.

Ces maisons enjambent parfois la rue, se projetant en encorbellements supportés par des consoles maçonnées. 

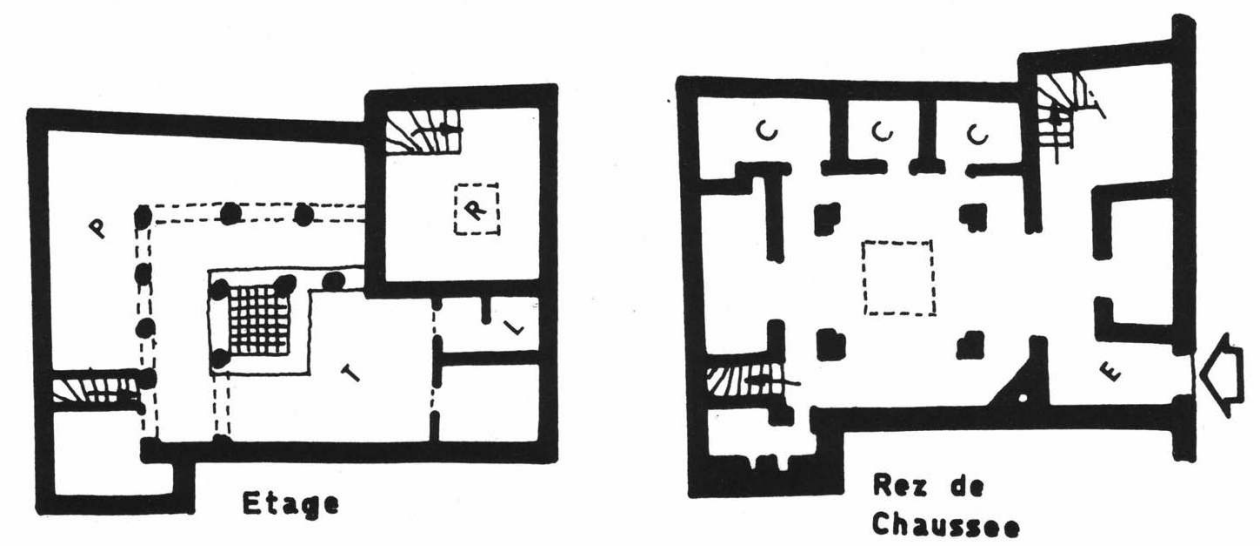

\section{La maison du Souf}

grande originalité de la maison du Souf est son mode de couverture faite de multiples coupoles obtenues par un mortier de gypse local mêlé de sable (tafzna), pétri et transmis de mains en mains par une chaîne d'aides jusqu'au maître d'œuvre. Celui-ci se tient sur un échafaudage en bordure des quatre murs de base préalablement construits et dont les angles ont été coupés par un système de trompes. Un mât, planté provisoirement au centre du carré, un clou fiché au sommet, tenant une ficelle, l'autre extrémité de cette ficelle passant entre le majeur et l'annulaire de la main du mu'allam, cela donnera, tendu, le rayon d'une demi-sphère, un nœud évitant le glissement entre les doigts du maitre-d'œuvre. Son travail consiste à déposer les boulettes de mortier et à lisser de la paume, corde tendue. La coupole est vite montée sans aucun secours de cintrage. Intérieurement, elle est parfaite; extérieurement, elle présente toutes les aspérités d'un nid d'hirondelles. Un badigeon de plâtre blanc sur l'intérieur achèvera le travail.

De plan, la maison s'organise autour d'une cour oblongue, accessible par une entrée en chicane. Les chambres rectangulaires sont généralement recouvertes de deux berceaux (demsa) accolés ; les coupoles (qubba) se dressant aux angles. Souvent, sur le côté sud, on peut voir des arcades formant un galerie appréciable aux heures chaudes de l'été. Mais il est fréquent de trouver, en guise de toiture de ces pièces allongées, des alignements de coupoles : trois, quatre, voire plus. Elle sont édifiées comme il a été dit, c'est-à-dire en divisant, intérieurement, l'espace rectangulaire en carrés par des arcades transversales.

Les chambres sont à usages multiples, au nord se tient l'étable abritant la ou les chèvres, l'âne ou le mulet; la cuisine se fait dans un angle ou au milieu de la cour, mais de préférence sous la galerie. Le mobilier se réduit à des nattes au sol, parfois des tapis et des couvertures de laine. Les murs de pierre à plâtre (rose des sables) sont percés de niches où l'on entrepose la lingerie et les objets d'usage courant. 


\section{La maison de Djerba}

29 Le menzel djerbien est une sorte de villa isolée dans la palmeraie, ayant parfois une allure militaire avec ses bastions en ghorfa et ses murs épais confortés par des arcsboutants (adjim).

Les pièces se distribuent autour d'une cour carrée ou barlongue, espace presque constamment occupé par les femmes. Sur trois côtés sont des chambres très allongées, le quatrième côté étant réservé aux communs : cuisine, latrines, magasins et entrée indirecte pratiquée souvent à l'intérieur d'une pièce carrée (sqîfa). Les chambres possèdent, à une de leurs extrémités, une banquette surélevée (dukkâna) qui sert de lit ; généralement, cet endroit est coiffé d'une coupole, un arc transversal délimitant l'alcôve et formant ainsi un carré de base. L'une de ces chambres est souvent couverte d'une pièce en étage, carrée, qui sert de poste d'observation ou de lieu de repos du maitre, et dont la silhouette trapue, en terrasse, se détache des couvertures en berceaux ou en coupoles. Cette chambre supérieure s'ouvre sur ses quatre faces mais parfois sur deux seulement (sud et est), on appelle ce belvédère kšǔk. Parfois, ces pièces hautes ne sont accessibles que par un escalier extérieur, en façade.

31 Les murs extérieurs offrent quelques rares ouvertures grillées en hauteur. Chaque chambre est pourvue d'un espace toilette et dispose de latrines à proximité. La porte unique est à deux battants massifs, bloquée, à l'intérieur, par un savant verrouillage en bois dur. De l'extérieur, on peut la fermer à l'aide d'une grossière serrure de fer. Les battants sont en palmier refendu (šannǔr).

32 Le mobilier traditionnel consiste en des coussins de laine, des nattes et des tapis et quelques coffres : des cordes d'alfa tendues soutiennent les vêtements. Le matériau de construction est un travertin local, calcaire coquillier de belle couleur orange, friable, liaisonné au mortier de chaux et de sable. A Guellala on fabrique un mortier de chaux et de cendres (provenant des fours de potiers), très solide. Le plâtre est obtenu à partir du gypse des carrières de Beni Diss, ou à partir des concrétions appelées roses des sables trouvées dans l'argile.

Les coupoles djerbiennes utilisent presque toujours des poteries spéciales tronconiques dont la petite base est plane tandis que la grande est courbe (tournée vers l'extérieur de la coupole).

Une des curiosités de la palmeraie est l'atelier traditionnel de tisserand, grande pièce voûtée en berceau avec frontons triangulaires aux deux extrémités. 


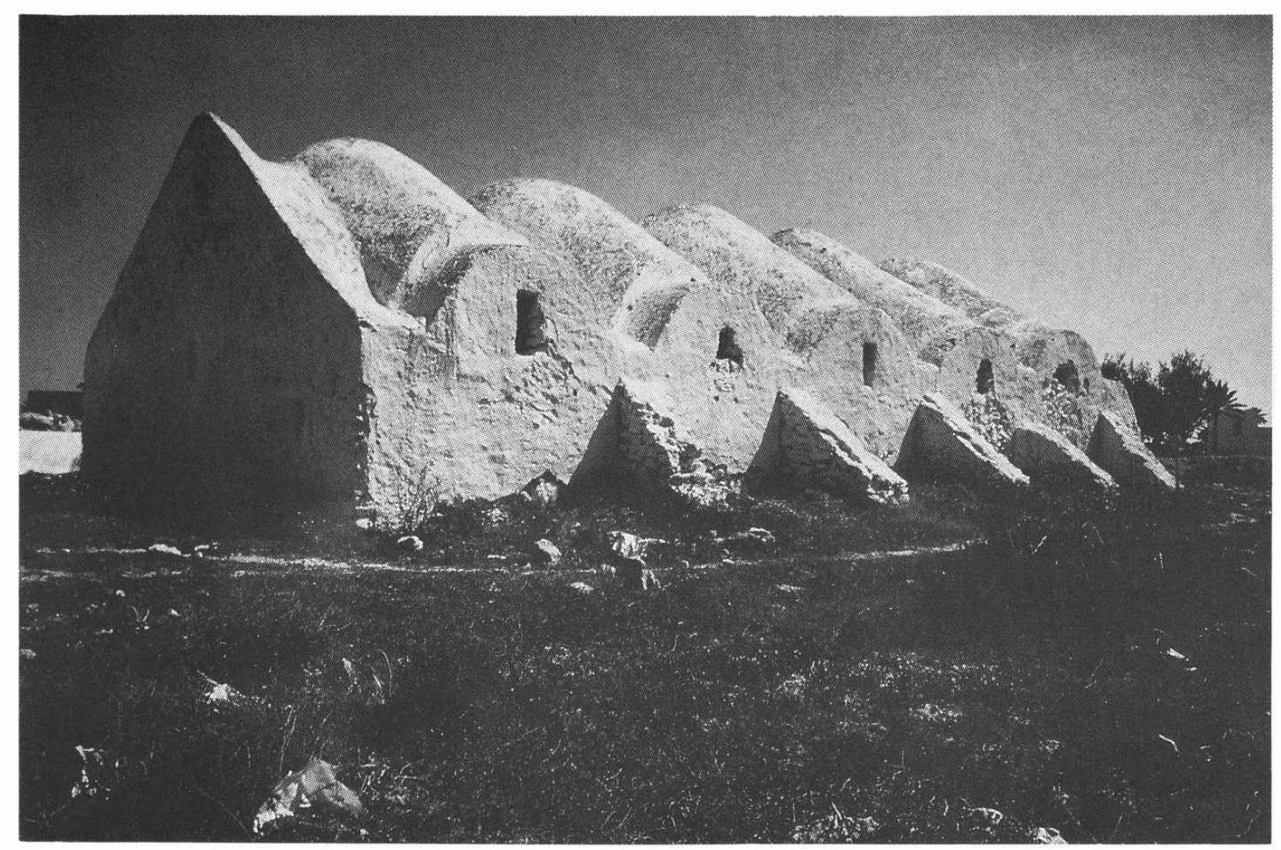

\section{Le maison oasienne de Tozeur (Tunisie)}

Tozeur offre, à coup sûr, une très grande originalité par le décor des façades extérieures des maisons. L'élément constructif exclusif est la brique modelée à la main et cuite au four.

Les dispositions générales procèdent, comme ailleurs, à partir d'une cour centrale, lieu de séjour et de distribution des pièces qui l'entourent. Comme ailleurs également, l'entrée (sqifa el-barraniya) est en chicane ; mais, ici, le vestibule-passage est aussi lieu de réunion et il dispose pour cela, face à la porte, de largues banquettes de terre (dukkāna) pratiquées dans des niches verticales en arcades reposant sur des piliers (arșa) de briques cuites dont l'abaque est en bois d'abricotier.

Cette première entrée communique avec une seconde, à angle droit (sqifa al-dahlaniya) qui ouvre sur le patio où le visiteur est saisi d'emblée par le décor profus des façades, obtenu par effets de briquetage. Ces ornements se trouvent à hauteur d'un second niveau où ils se composent de panneaux et de registres qui ne sont pas sans évoquer des tissages décorés, des tentures d'apparat: alignements de chevrons ou résilles, alignements ou superpositions de carrés sur pointe, de polygones plus complexes, que trouent des ouvertures en archères. 


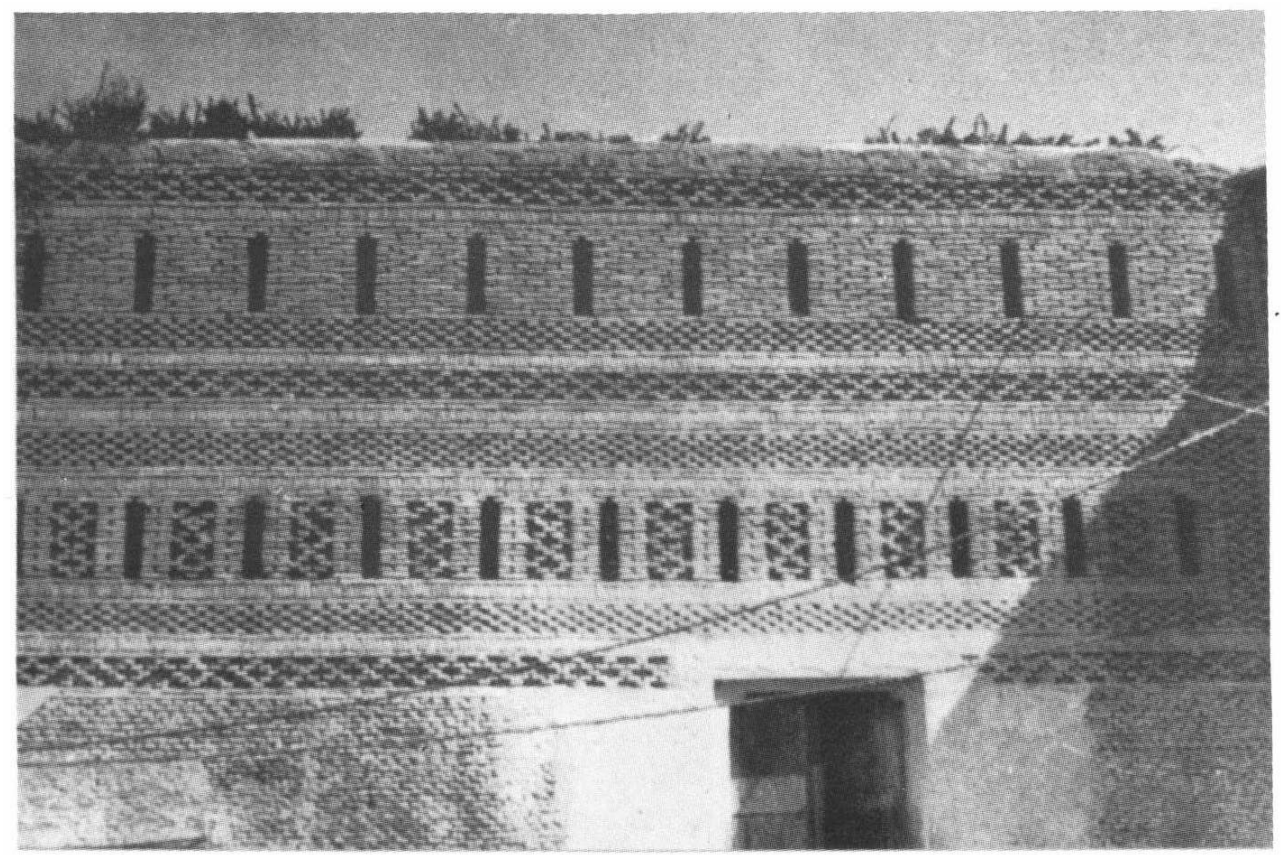

38 Au rez-de-chaussée, on trouve, au centre de la cour, une fosse à ordures. Sur un côté, généralement à droite en entrant, sont les dépendances : écuries, latrines, cuisine. Sur les autres côtés s'étendent les chambres rectangulaires parfois très longues (de 12 à 25 $\mathrm{m}$ ), prolongées d'une alcôve carrée ; l'entrée de cette alcôve est souvent d'une grande beauté avec sa porte en arc de plein cintre flanquée de panneaux de briques appareillées en carrés sur pointe superposés. Au-dessus s'ouvrent deux baies jumelées, en arcs de plein cintre, protégées par des balustrades de bois ouvré. C'est le mahzzen.

Dans la pièce principale, des poutres transversales, à diverses hauteurs, et des cordes tendues, servent à étendre le linge ou à suspendre des régimes de dattes tandis que le sol supporte d'énormes poteries modelées, réserves de dattes.

40 L'escalier de la cour conduit à l'étage qui, parfois, comporte une galerie sur l'un de ses côtés, ouverte sur la cour par des arcades sur piliers.

On ne distingue aucun accès aux terrasses composées de troncs de palmiers et de stippes de palmes supports d'un damage de pierraille et de terre.

Il arrive fréquemment qu'un corps de logis enjambe la ruelle sur laquelle il s'ouvre en fenêtre à jalousie, ornées d'un décor de briquetage au-dessus duquel se projette une gargouille faite d'une branche d'arbre évidée.

43 Étranges en vérité ces belles demeures de Tozeur qui ne sont pas sans rapport avec celles de la Tihama yéménite (Zabid en particulier). Simple coïncidence sans doute.

44 Tout le vocabulaire est arabe, la population étant bédouine mêlé d'un fond berbère, mais on retrouve, dans ces décors géométriques des façades, des compositions qui ne manquent pas d'évoquer les décors des poteries modelées, des tissages berbères, voire des coffres kabyles. Sans doute sont-ils les vestiges d'un art ancestral qui n'a pas oublié son lointain passé, celui-là même déjà évoqué à Sadrata. 


\section{Les greniers-citadelles du Maroc} horizontales alignées, confectionnées à l'aide de pierres plates à l'intérieur desquelles on introduisait des couffes d'alfa; une dalle assurait la fermeture. Ailleurs, les constructions en pierres sèches comprenaient plusieurs étages accessibles par des dalles saillantes en façade et desservies par des ruelles étroites; les cellules étaient bouchées par une porte verrouillée (Toumliline, Tasguinnt, Ougeuzmir, etc.) parfois surmontées d'un décor chevronné. Quelques ensembles constituent de véritables forteresses tel Tagadirt Dou-Igadirt, Agadir n-Iseryine, I yerm n Aït Elhassade Ou Daoud, Ouatabe, El qelâa $\mathrm{n}$ Imgounn, avec ses tours rondes plus rares que les tours carrées. Dans le Haut Atlas, ces ensembles, construits en pisé, offrent de beaux décors de briques de terre sèche (Anemiter, El-Qalâa n-Imgunn uātab) et il est assez difficile souvent de distinguer le grenier de l'habitat. On notera, à Iboukhennane, un très curieux appareil de galets alignés sur un ou deux lits, parfois bordés de pierres plates, alternant avec des appareils en épis.

\section{Aurès}

Les gal'a ou greniers-citadelles, autrefois répandus dans la montagne, ont disparu ou sont en voie de disparition ; très curieuse était la gal'a de Béniane, construite en terre, sur des formes arrondies couronnées par une superposition de disques avec planchers et terrasses débordant sur l'extérieur. Chacun de ces étages était percé de portes donnant sur des magasins. On y accédait par des escaliers extérieurs faits de rondins de bois fichés dans la maçonnerie.

\section{Les greniers-citadelles du Sud tunisien}

Les ensembles le plus connus étaient les ksours de Médenine, avec leurs greniers (yorfa) superposés, le dernier étage étant couvert de berceaux parallèles. Une grande partie de ces constructions a disparu après l'indépendance tunisienne. Il s'agissait de magasins individuels en étages. Aux rez-de-chaussée habitaient les gardiens ainsi que les animaux: chameaux, ânes ou mulets. On comptait, jadis, jusqu'à 6000 ghorfas construites en pierraille noyée dans un mortier d'argile. Un escalier extérieur

Encyclopédie berbère, 6 | 1989 
desservait jusqu'à quatre niveaux, voire plus, de cellules fermées par des portes cadenassées. On ensilait là des céréales, des dattes, les produits des troupeaux. L'ensemble formait une véritable ville. Outre Médenine, il faut citer Métameur, au centre d'une maigre palmeraie, avec sa mosquée, sa grande place autour de laquelle se serraient les yorfa et quelques ksours isolés dans la haute Jefâra ainsi que quelques ksours de plaine. Assez semblables étaient les greniers dits kasbah (qaṣba), bâtisses de pierre de forme carrée que l'on trouvait au Djebel Abiodh, les Kasbah 'Abd al-Jouad, près de Maztouiya (vallée de l'oued Zondag), comprenant deux grandes pièces voûtées au rez-de-chaussée et, à l'étage, deux ghorfa.

L'architecture berbère est une réalité aussi évidente que l'est la langue berbère. Comme elle, elle connaît ses provincialismes ; comme elle, elle n'est pas restée hermétique à des apports étrangers, mais comme elle, elle présente suffisamment d'unité pour qu'on la distingue.

Sans doute est-elle mieux ancrée dans des régions restées fidèles à la vieille civilisation locale : la montagne ou le Sahara; mais ailleurs et surtout aux confins de ces zones à forte densité berbère, aux endroits où l'arabisation s'est effectuée depuis plusieurs siècles, il ne faut pas chercher bien loin pour retrouver le vieux fond berbère. Il s'exprime alors surtout dans les coutumes et dans les techniques, dans la poterie modelée ou dans les tissages, par exemple, et certainement encore dans l'architecture.

Ghorfa de Médenine (photo G. Camps).

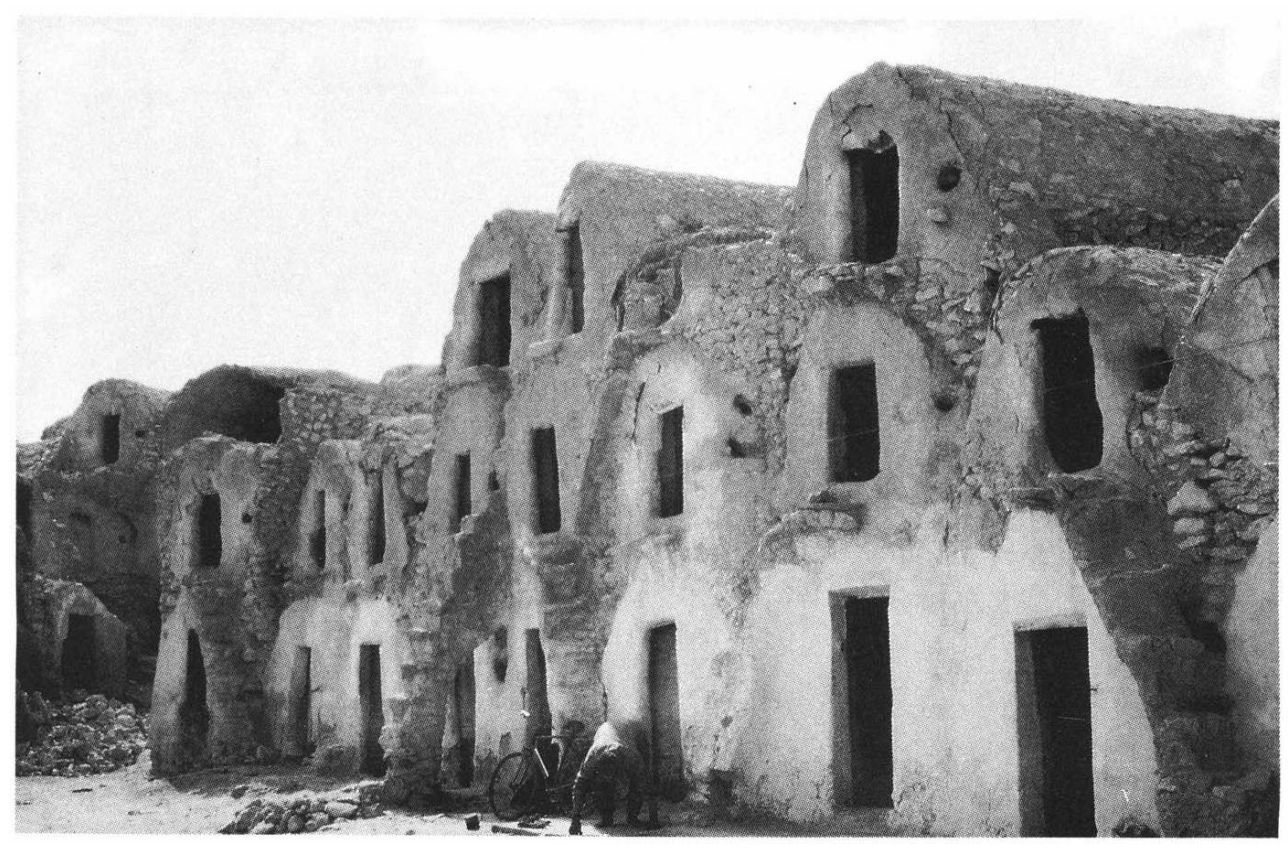




\section{BIBLIOGRAPHIE}

BOURDIEU P., Sociologie de l'Algérie, coll. « Que Sais-je ? ", nº 80.

BONETE Y., « Contribution à l'étude de l'habitat au Mzab », Cahiers des arts et techniques d'Afrique du Nord, t. 5, 1959.

BONETE Y., « La culture mozabite », Documents algériens, série monographie, n² 23, 1958,

GAUDRY M., La femme chaouïa de l'Aurès, Paris, 1929.

GAUDRY M., La société féminine au Djebel Amour et au Ksel, Alger, 1961.

GAILlARD Cpte, « Deux oasis du Bani Tata et Tissint, sanctuaires et marabouts », Arch. S.H. A., 1949.

GAILlARD Cpte, «Étude sur les Ksours et ses ruines », Arch. S. H. A., 1949.

JACQUES-MEUNIÉ D., Greniers-citadelles du Maroc, Paris, Arts et métiers graphiques, 1951.

JACQUES-MEUNIÉ D., Architecture et habitats du Dadès (Maroc présaharien), Paris, Klincksieck, 1962.

JACQUES-MEUNIÉ D., Cités anciennes de Mauritanie, Paris, 1961.

JACQUES-MEUNIÉ D., Le Maroc saharien des origines à 1670, 2 vol. , Paris, 1982.

LOUIS A., Tunisie du sud, Ksars et villages de crêtes, Paris, 1975.

MARTIN A.G.P., Les oasis sahariennes (Gourara, Touat, Tidikelt), Paris, 1908.

MERCIER M., La civilisation urbaine au Mzab, Alger, 1922.

MERCIER M., Ghardaïa la mystérieuse, Alger, 1932.

PARIS A., Documents d'architecture berbère au sud de Marrakech, Rabat, 1925.

ROCHE M., Le Mzab, architecture ibadite, Paris, 1970.

TERRASSE H., Kasbas berbères de l'Atlas et des oasis, Paris, 1938.

TLATLI S., Djerba et les Djerbiens, Tunis, 1942.

INDEX

Mots-clés : Architecture, Maghreb, Religion, Sociologie 